\title{
On the Gravitational Constant and Radius in Spherically Symmetric Problem of General Relativity
}

\author{
Valery Vasiliev $^{1} \&$ Leonid Fedorov ${ }^{1}$ \\ ${ }^{1}$ Institute of Problems of Mechanics, Russian Academy of Sciences, 101 Vernadskogo, Moscow, Russia \\ Correspondence: Valery Vasiliev, Institute of Problems of Mechanics, Russian Academy of Sciences, 101 \\ Vernadskogo, Moscow 119526, Russia. Tel: 7-916-607-1281. E-mail: vvvas@dol.ru
}

Received: March 30, 2013

Accepted: April 26, 2013 Online Published: May 5, 2013

doi:10.5539/apr.v5n3p116

URL: http://dx.doi.org/10.5539/apr.v5n3p116

\begin{abstract}
The paper is concerned with the gravitational constant in the General Theory of Relativity (GTR) which is traditionally determined under the condition of asymptotic reduction of GTR equations to Newton gravitation equation for low intensity gravitation field. As shown in the paper, for spherically symmetric static GTR problem, the traditional gravitational constant is accordingly valid for low intensity gravitation field only and should be generalized for gravitation fields with high intensities. The same conclusion is made for the gravitational (Schwarzschild) radius.
\end{abstract}

Keywords: general relativity, spherically symmetric problem, gravitational constant, gravitational radius

\section{Introduction}

The classical Newton's law of gravitation, according to which the gravitational force acting between two bodies with masses $m_{1}$ and $m_{2}$ located at a distance $r$ from each other is specified as $F=G m_{1} m_{2} / r^{2}$ and includes the universal constant $G$ originally measured with the aid of a torsion balance by Cavendish in 1798. Refined value of this constant $\left(\mathrm{G}=6.693 \times 10^{-11} \mathrm{Nm}^{2} / \mathrm{kg}^{2}\right)$ has been found by Fixler, Foster, McGuirk and Kasevich (2007) from the atom interferometer measurement. Within the framework of the classical gravitation theory, the gravitational potential $\phi$ satisfies the following Poisson's equation:

$$
\Delta \phi=4 \pi G \rho
$$

which includes the universal constant $G$ and the matter density $\rho$.

In GTR, the energy tensor $T_{i j}$ is associated with the Ricci curvature tensor $R_{i g}$ through Einstein equation

$$
\chi T_{i j}=R_{i j}-\frac{1}{2} R g_{i j}
$$

which includes the GTR gravitational constant $\chi$. To express $\chi$ in terms of $G$ the gravitation field with low intensity for which the metric coefficients $g_{i j}$ are close to the corresponding coefficients of the Euclidean space is considered. The only one remaining Equation (2) for $i=j=4$ is reduced to Equation (1) and the following relationship between $\chi$ and $G$ is established:

$$
\chi=8 \pi G / c^{4}
$$

in which $c$ is the velocity of light. Traditionally, Equation (3) is treated as the universal equation for $\chi$ which does not depend on the intensity of the gravitation field. However in the strict sense, the derivation of Equation (3) is valid only for the gravitation fields with low intensity and we can suppose that in the general case, the expression for $\chi$ can be different from Equation (3) which provides only the asymptotic value of this constant valid for gravitation fields with relatively low intensities. The paper provides the corresponding expression for the spherically symmetric GTR problem.

\section{Equations of the Classical Gravitation Theory}

Within the framework of the classical gravitation theory, the gravitational potential $\phi$ which governs the spherically symmetric gravitation field induced by a solid sphere with radius $R$ and density $\rho$ satisfies the following Poisson's equation which is a particular case of Equation (1): 


$$
\frac{1}{r^{2}}\left(r^{2} \phi^{\prime}\right)^{\prime}=4 \pi G \rho
$$

in which $r$ is the radial coordinate, $G$ is the classical gravitational constant and $(\ldots)^{\prime}=d(\ldots) / d r$. The solutions of Equation (1) for the internal space for which $0 \leq r \leq R$ and $\rho=$ const (index " $i$ ") and the external space for which $R \leq r<\infty$ and $\rho=0$ (index "e") are

$$
\phi_{i}^{\prime}=(4 / 3) \pi G \rho r, \phi_{e}^{\prime}=C_{1} / r^{2}
$$

Using the boundary condition $\phi_{i}^{\prime}(R)=\phi_{e}^{\prime}(R)$ to determine the constant $C_{1}$, integrating and neglecting the integration constant which is not important for the potential function, we get for the potential of the external space

$$
\phi_{e}=-\gamma m / r
$$

where

$$
m=m_{0}=(4 / 3) \pi \rho R^{3}
$$

is the sphere mass in the Euclidean space.

Introduce the so-called gravitational radius $r_{g}^{0}$ which was found by Michell in 1783 and Laplace in 1796 (Thorne, 1994) from the following equation for the escape velocity:

$$
v=\sqrt{2 m_{0} G / R}
$$

Taking $v=c$ and $R=r_{g}^{0}$, we get

$$
r_{g}^{0}=2 m_{0} G / c^{2}
$$

which is the radius of the sphere for which the escape velocity is equal to the velocity of light $c$.

\section{Equations of the General Theory of Relativity}

Consider a GTR spherically symmetric problem for which the line element of the semi-Riemannian space induced by the gravitational field can be written as (Synge, 1960)

$$
d s^{2}=g d r^{2}+r^{2}\left(d \theta^{2}+\sin ^{2} \phi d \phi^{2}\right)-h c^{2} d t^{2}
$$

where $g(r)$ and $h(r)$ are the coefficients of the metric tensor. The components of the energy tensor for the static problem are (for the problem under study the mixed components which coincide with the corresponding physical components are used)

$$
T_{1}^{1}=\sigma_{r}, T_{2}^{2}=T_{3}^{3}=\sigma_{\theta}, T_{4}^{4}=\rho c^{2}
$$

Here, $\sigma_{r}$ and $\sigma_{\theta}$ are the radial and the circumferential stresses induced by the gravitational field. The energy tensor, $T_{i}^{j}$, must satisfy the following conservation equation (Synge, 1960):

$$
\left(T_{1}^{1}\right)^{\prime}-\frac{2}{r}\left(T_{2}^{2}-T_{1}^{1}\right)+\frac{h^{\prime}}{2 h}\left(T_{1}^{1}-T_{4}^{4}\right)=0
$$

According to GTR, Equation (11) is satisfied identically if the energy tensor is expressed in terms of the Einstein tensor $G_{i}^{j}$ as

$$
\begin{gathered}
\chi T_{1}^{1}=G_{1}^{1}=-\frac{1}{g}\left(\frac{h^{\prime}}{r h}+\frac{1}{r^{2}}\right)+\frac{1}{r^{2}} \\
\chi T_{2}^{2}=\chi T_{3}^{3}=G_{2}^{2}=-\frac{1}{2 g}\left[\frac{h^{\prime \prime}}{h}-\frac{1}{2}\left(\frac{h^{\prime}}{h}\right)^{2}+\frac{1}{r}\left(\frac{h^{\prime}}{h}-\frac{g^{\prime}}{g}\right)-\frac{g^{\prime} h^{\prime}}{2 g h}\right]
\end{gathered}
$$




$$
\chi T_{4}^{4}=G_{4}^{4}=-\frac{1}{g}\left(\frac{1}{r^{2}}-\frac{g^{\prime}}{r g}\right)+\frac{1}{r^{2}}
$$

in which $\chi$ is the GTR gravitational constant. Equations (12)-(14) present the explicit form of Equation (2) for spherically symmetric problem. Because substitution of Equations (12)-(14) in Equation (11) satisfies this equation identically, only three of four Equations (11)-(14) are mutually independent. Traditionally (Synge, 1960), the simplest set of equations which is used for analysis includes Equations (11), (12) and (14). The remaining Equation (13), is satisfied identically. Substituting Equation (10) in Equations (11), (12) and (14), we arrive at the following final set:

$$
\begin{gathered}
\sigma_{r}^{\prime}-\frac{2}{r}\left(\sigma_{\theta}-\sigma_{r}\right)+\frac{h^{\prime}}{2 h}\left(\sigma_{r}-\rho c^{2}\right)=0 \\
\chi \sigma_{r}=-\frac{1}{r g}\left(\frac{h^{\prime}}{h}+\frac{1}{r}\right)+\frac{1}{r^{2}} \\
\chi \rho c^{2}=\frac{1}{r^{2}}\left(r-\frac{r}{g}\right)^{\prime}
\end{gathered}
$$

The boundary conditions of the problem

$$
\sigma_{r}(r=0)=\sigma_{\theta}(r=0), \sigma_{r}(r=R)=0
$$

should be supplemented with the regularity condition at the origin $r=0$, the compatibility conditions of the metric tensor for the internal and the external space at $r=R$ and the asymptotic conditions at $r \rightarrow \infty$.

\section{GTR Gravitational Constant}

As follows from Introduction, the GTR gravitational constant $\chi$ which enters Equations (16) and (17) is traditionally expressed in term of the gravitational constant $G$ in Equation (4) of the classical gravitation theory. In application to the spherically symmetric problem, consider the semi-Riemannian space for which the coefficients of the line element form in Equation (9) are close to the coefficients of the corresponding Euclidean space, i.e.,

$$
g=1+f_{r}(r), \quad h=1+f_{t}(r)
$$

where $|f|<<1$. Performing linearization of Equations (15)-(17) with respect to $f$, we arrive at

$$
\begin{aligned}
& r \sigma_{r}^{\prime}-2\left(\sigma_{\theta}-\sigma_{r}\right)=0 \\
& \chi_{0} \sigma_{r}=\frac{1}{r^{2}}\left(f_{r}-r f_{t}^{\prime}\right) \\
& \chi_{0} \rho c^{2}=\frac{1}{r^{2}}\left(r f_{r}\right)^{\prime}
\end{aligned}
$$

We introduce here the GTR gravitational constant $\chi_{0}$ which corresponds to the linearized equations. As shown further, this constant, in general, does not coincide with $\chi$ in Equations (16) and (17).

Expressing $f_{r}$ in terms of $f_{t}$ and $\sigma_{r}$ with the aid of Equation (21) and substituting the result in Equation (22), we get

$$
\frac{1}{r^{2}}\left(r^{2} f_{t}^{\prime}\right)^{\prime}=\chi_{0}\left(\rho c^{2}-3 \sigma_{r}-r \sigma_{r}^{\prime}\right)
$$

Expressing $\sigma_{r}^{\prime}$ from Equation (20), we can reduce this equation to

$$
\frac{1}{r^{2}}\left(r^{2} f_{t}^{\prime}\right)^{\prime}=\chi_{0} \rho c^{2}\left(1-\frac{\sigma_{0}}{\rho c^{2}}\right)
$$

where $\sigma_{0}=\sigma_{r}+2 \sigma_{\theta}$ is the first invariant of the stress tensor for the sphere under consideration. To compare Equation (23) with Equation (4) of the classical gravitation theory, we need to link the function $f_{t}(r)$ with the gravitational potential $\phi(r)$. For this purpose, the trajectory of a small object in a gravitational field induced by a solid is traditionally considered. In the classical theory, the trajectory is governed by the gravitational force 
with potential $\phi(r)$, whereas in GTR, it coincides with the geodesic line of the semi-Riemannian space whose curvature depends on function $f_{t}(r)$. Because the trajectories must be the same, the following relationship between the functions is valid (Weinberg, 1972):

$$
f_{t}(r)=2 \phi(r) / c^{2}
$$

Substituting this result in Equation (23), we arrive at

$$
\frac{1}{r^{2}}\left(r^{2} \phi^{\prime}\right)^{\prime}=\frac{1}{2} \chi_{0} \rho^{*} c^{4}
$$

where

$$
\rho^{*}=\rho(1-\alpha), \alpha=2 \sigma_{0} / \rho c^{2}
$$

Note that the right-hand part of Equation (24) which is derived here for the spherically symmetric problem includes the reduced density $\rho^{*}$. In the general case, if (in contrast to the traditional derivation) the stresses are not neglected, the resulting Poisson's equation similar to Equation (1) also includes $\rho^{*}$ (Vasiliev \& Fedorov, 2003). Thus, tensile stresses reduce the effective density and hence, the gravitational potential of a solid, whereas compressive stresses (i.e., negative values of the stress invariant) increase the density and the gravitational potential. Tensile stresses can occur, for example, in steadily spinning solids. The maximum $\sigma_{0}$ value can be reached today in composite flywheels of uniform strength reinforced with high-strength carbon fibers (Vasiliev, 1993). For such flywheels, $\sigma_{0}=3600 M P a, \rho=1550 \mathrm{~kg} / \mathrm{m}^{3}$ and $\alpha=0.52 \cdot 10^{-10}$. Thus, the stress effect is small and for real structural materials we can neglect $\alpha$ in comparison with unity taking $\rho^{*}=\rho$ in Equation (24). Then, this equation coincides with Equation (4) if

$$
\chi_{0}=8 \pi G / c^{4}
$$

which is the traditional expression in Equation (3) for the GTR gravitational constant.

\section{Solution of the GTR Equations for a Spherically Symmetric Space}

Consider the traditional solutions of Equations (15)-(17) corresponding to the external $(R \leq r<\infty)$ and the internal $(0 \leq r \leq R)$ spaces of the solid sphere with radius $R$ (Schwarzschild, 1916).

For the empty external space, $\rho=0$ and $\sigma_{\mathrm{r}}=\sigma_{\theta}=0$. Then, Equation (15) is satisfied identically and the remaining Equations (16) and (17) can be reduced to

$$
\frac{h_{e}^{\prime}}{h_{e}}=\frac{1}{r}\left(g_{e}-1\right),\left(r-\frac{r}{g_{e}}\right)^{\prime}=0
$$

Integration of Equations (27) yields

$$
g_{e}=\frac{1}{1+C_{2} / r}, \quad h_{e}=C_{3}\left(1+\frac{C_{2}}{r}\right)
$$

Determine the integration constants $C_{2}$ and $C_{3}$. For $r \rightarrow \infty$, Equations (28) must reduce to the solution of the classical gravitation theory (Landau \& Lifshitz, 1962), i.e., to

$$
g_{e}^{0}=1-\frac{2 \phi_{e}}{c^{2}}, h_{e}^{0}=1+\frac{2 \phi_{e}}{c^{2}}
$$

where the gravitational potential $\phi_{e}$ is specified by Equation (6). Then, Equations (28) become

$$
g_{e}=\frac{1}{1-r_{g}^{0} / r}, \quad h_{e}=1-\frac{r_{g}^{0}}{r}
$$

where $r_{g}^{0}$ is the gravitational radius specified by Equation (8). As follows from Equations (29) the metric coefficient can become singular if the radius $r$ reaches the value $r_{g}^{0}$ which is referred to as the radius of the Black Hole event horizon (Frolov \& Zelnikov, 2011). It does not look natural that Equations (29) corresponding to GTR include the radius $r_{g}^{0}$ which is specified by Equation (8) corresponding to the classical gravitation 
theory.

For the internal space, $\rho=$ const and integration of Equation (17) in which $\chi=\chi_{0}$ in accordance with Equation (26) yields

$$
g_{i}=\frac{1}{1-\frac{\chi_{0}}{3} \rho c^{2} r^{2}+\frac{C_{4}}{r}}
$$

From the traditional regularity condition it follows that $C_{4}=0$ (Misner, Thorne, \& Wheeler, 1973). Apply the compatibility condition for the metric coefficient at the sphere surface $r=R$, according to which $g_{e}(R)=g_{i}(R)$. Using Equations (29) and (30), we arrive at the following relationship:

$$
\frac{\chi_{0}}{3} \rho c^{2} R^{3}=r_{g}^{0}
$$

Then, Equation (30) takes the following final form:

$$
g_{i}=\frac{1}{1-\frac{r_{g}^{0} r^{2}}{R^{3}}}
$$

Substituting $r_{g}^{0}$ from Equation (8) in Equation (31) and taking into account that $m_{0}$ is specified by Equation (7), we can find $\chi_{0}=8 \pi \mathrm{G} / \mathrm{c}^{4}$ which coincides with Equation (26).

Note that if $r_{g}^{0}$ is much smaller than $R$, we can neglect the terms with $r_{g}^{0} / R$ in comparison with unity in Equations (29) and (32) and the metric coefficients reduce to the corresponding coefficients of the Euclidean space for which the sphere mass $m_{0}$ is specified by Equation (7). However, if the space inside the sphere is not Euclidean, the sphere mass is not equal to $m_{0}$. Calculating the sphere mass corresponding to the Riemannian space with the metric coefficient specified by Equation (32), we arrive at the following expression:

$$
m=2 \rho \int_{0}^{2 \pi} d \theta \int_{0}^{\pi} \sin \phi d \phi \int_{0}^{R} \sqrt{g_{i}} r^{2} d r=\frac{2 \pi}{\bar{r}_{g}} \rho R^{3}\left(\frac{1}{\sqrt{r_{g}}} \sin ^{-1} \sqrt{r_{g}^{-0}}-\sqrt{1-r_{g}}\right)
$$

in which

$$
\bar{r}_{g}^{0}=r_{g}^{0} / R
$$

Decomposing the right-hand part of Equation (33) into the power series and using Equation (7) for $m_{0}$, we get

$$
m=m_{0}\left[1+\frac{3}{10} r_{g}^{-0}+\frac{9}{56}\left(r_{g}^{-0}\right)^{2}+\ldots\right]
$$

This equation coincides with Equation (7) only for the Euclidean space for which $\vec{r}_{g}=0$. In the general case, $m \neq m_{0}$ which means that either the space inside the sphere is not Riemannian (Vasiliev \& Fedorov, 2013) or the theory is not consistent. Indeed, it looks natural that if the GTR equations are valid, the potential function $\phi_{e}$ in Equation (6) which governs the gravitational field at a distance from the sphere should depend on the actual mass of the sphere $m$ specified by Equation (33).

\section{New Expressions for the Gravitational Radius and Constant}

Because $r_{g}^{0}$ and $\chi_{0}$ correspond to the Euclidean space to which the Riemannian space is reduced for gravitation fields with low intensities, assume that these constants are only the asymptotic values of the corresponding GTR constants. In the general case, the sphere mass $m$ corresponds to the Riemannian space and Equation (8) for the gravitational radius can be generalized as

$$
r_{g}=2 m \gamma / c^{2}
$$

Repeating the derivation of Equations (29) and (32), we arrive at the following metric coefficients for the external and the internal spaces: 


$$
g_{e}=\frac{1}{1-\frac{\bar{r}_{g}}{\bar{r}}}, \quad g_{i}=\frac{1}{1-\bar{r}_{g} \bar{r}^{2}}
$$

in which

$$
\bar{r}_{g}=r_{g} / R, \bar{r}=r / R
$$

Note the second equation of Equations (37) exists under the condition that the following relationship which generalizes Equation (31) is valid:

$$
\frac{\chi}{3} \rho c^{2} R^{3}=r_{g}
$$

Here, $r_{g}$ is specified by Equation (36) and $\chi$ is the new GTR gravitational constant.

Now, the sphere mass which is specified by the left-hand part of Equation (33) becomes

$$
m=\frac{2 \pi}{\bar{r}_{g}} \rho R^{3}\left(\frac{1}{\sqrt{\bar{r}_{g}}} \sin ^{-1} \sqrt{\bar{r}_{g}}-\sqrt{1-\bar{r}_{g}}\right)
$$

Substituting $m$ from Equation (40) in Equation (36), we get the following equation for the gravitational radius:

$$
\bar{r}_{g}=\frac{3 \bar{r}_{g}}{2 \bar{r}_{g}}\left(\frac{1}{\sqrt{\bar{r}_{g}}} \sin ^{-1} \sqrt{\bar{r}_{g}}-\sqrt{1-\bar{r}_{g}}\right)
$$

in which $r_{g}^{0}$ is the traditional gravitational radius specified by Equation (8). Approximate version of this equation corresponding to Equation (35) is

$$
\bar{r}_{g}=\bar{r}_{g}^{0}\left[1+\frac{3}{10} \bar{r}_{g}+\frac{9}{56} \bar{r}_{g}^{2}+\ldots\right]
$$

It looks natural that the derived expression for the gravitational radius in GTR does not coincide with Equation (8) following from the classical gravitation theory.

Using Equation (39), we can determine now the corresponding value of the gravitational constant, i.e.,

$$
\chi=\frac{3 \chi_{0}}{2 \bar{r}_{g}}\left(\frac{1}{\sqrt{\bar{r}_{g}}} \sin ^{-1} \sqrt{\bar{r}_{g}}-\sqrt{1-\bar{r}_{g}}\right)
$$

Or approximately

$$
\chi=\chi_{0}\left[1+\frac{3}{10} \bar{r}_{g}+\frac{9}{56} \bar{r}_{g}^{2}+\ldots\right]
$$

Where $\chi_{0}$ is the traditional GTR gravitational constant specified by Equation (26). For $r_{g} \rightarrow 0$, we have $\chi \rightarrow \chi_{0}$.

Note that the metric coefficients in Equations (37) are formally the same that follow from the traditional Schwarzchild solution, but the gravitational radius $r_{g}$ is different from the Schwarzchild radius $r_{g}^{o}$ and, in accordance with Equation (42), coincides with it for gravitation fields with relatively low intensities.

Dependences between the ratios $\bar{r}_{g} / \bar{r}_{g}, \chi / \chi_{0}$ and $\bar{r}_{g}^{0}$ plotted in accordance with Equations (41)-(44) are presented in Figure 1. As can be seen, $r_{g}$ is close to the traditional value $r_{g}^{0}$ only for low $r_{g}^{0}$ values. The maximum possible value of $\bar{r}_{g}$ is 1 , whereas the corresponding $\bar{r}_{g}$ value is $4 / 3 \pi$. As follows from Figure 1 , the normalized traditional gravitational radius reaches the maximum value 0.544 which means that that sphere radius $R$ cannot be lower than $1.838 r_{g}^{0}$. 


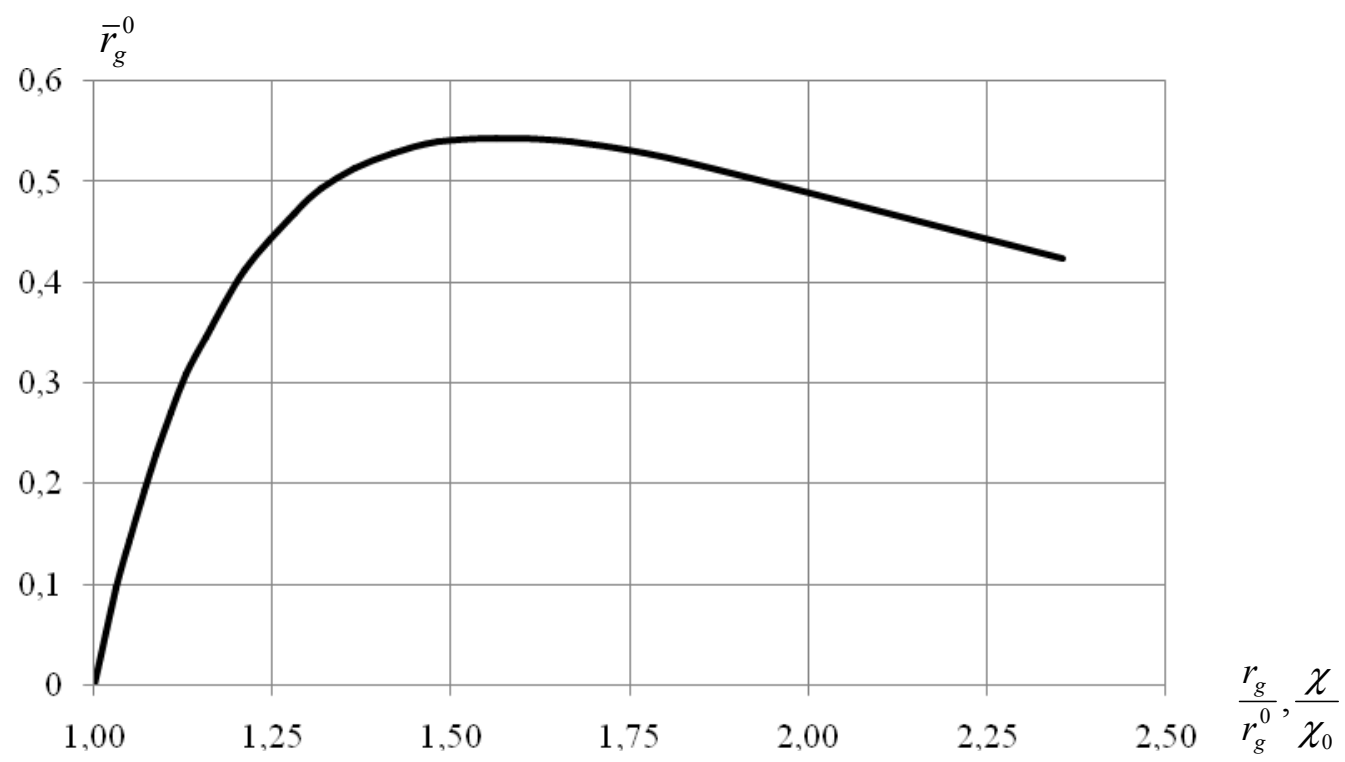

Figure 1. Dependences between the rarios $r_{g} / r_{g}^{0}, \chi / \chi_{0}$ and the traditional gravitational radius $r_{g}^{0}$

\section{Conclusion}

As follows from the foregoing analysis, the traditional gravitational constant $\chi_{0}$ which is obtained matching the linearized GTR equations with the corresponding equations of the classical gravitation theory is not universal and can be treated as the asymptotic value valid for gravitation fields with low intensity. In the general case, the GTR gravitational constant $\chi$ depends not only on the classical gravitational constant $G$, but also on the mass of a body inducing the gravitation. Respectively, the gravitational radius $r_{g}$, in the general case, does not coincide with the traditional Schwarzschild radius. For spherically symmetric static problem of General Relativity, close-form analytical solutions which specify the GTR gravitational constant and radius are obtained. If the body mass $m$ is such that the parameter $2 m G / c^{2}$ is much less than unity, the obtained expressions for $\chi$ and $r_{g}$ provide the traditional results.

\section{References}

Frolov, V. P., \& Zelnikov, A. (2011). Introduction to Black Hole Physics. Oxford: Oxford University Press.

Fixler, J. B., Foster, G. T., McGuirk, J. M., \& Kasevich, M. A. (2007). Atom interferometer measurement of the Newtonian constant of gravity. Science, 315(5808), 74-77. http://dx.doi.org/10.1126\%2Fscience.1135459

Landau, L. D., \& Lifshitz, E. M. (1962). Field Theory. Moscow: Nauka (in Russian).

Misner, S. W., Thorne, K. S., \& Wheeler, J. A. (1973). Gravitation. San Francisco: W. H. Freeman and Company.

Schwarzschild, K. (1916a). Uber das Gravitationsfeld eines Massenpunkies nach der Einsteinshen Theorie. Sitzungsberichte der Deutschen Akademie der Wissenschaften zu Berlin, Klasse fur Mathematik, Physik, und Technik. 189.

Schwarzschild, K. (1916b). Uber das Gravitationsfeld einer Kugel aus incompressibler Flussigkeit nach der Einsteinschen Theorie. Sitzungsberichte der Deutschen Arademie der Wissenschaften zu Berlin, Klasse fur Mathematik, Physic, und Technik. 424.

Synge, J. L. (1960). Relativity: the General Theory. Amsterdam: North Holland.

Vasiliev, V. V. (1993). Mechanics of Composite Structures. Washington: Taylor \& Francis.

Vasiliev, V. V., \& Fedorov, L. V. (2013). The problem of general relativity for an orthotropic solid sphere. Applied Physics Research, 5(1), 115-125. http://dx.doi.org/10.5539/apr.v5n1p115

Weinberg, S. (1972). Gravitation and Cosmology. New York: Willey. 